\title{
Exosomes Derived From Bone Marrow Mesenchymal Stem Cells Inhibit Complement Activation In Rats With Spinal Cord Injury
}

This article was published in the following Dove Press journal:

Drug Design, Development and Therapy

\author{
Chuanliang Zhao $\mathbb{D}^{1-3}$ \\ Xin Zhou ${ }^{1,2}$ \\ Jie Qiu ${ }^{1,2}$ \\ Danqing $\mathrm{Xin}^{2}$ \\ Tingting $\mathrm{Li}^{2}$ \\ Xili $\mathrm{Chu}^{2}$ \\ Hongtao Yuan ${ }^{2}$ \\ Haifeng Wang' \\ Zhen Wang ${ }^{2}$ \\ Dachuan Wang' \\ 'Department of Spinal Surgery, Shandong \\ Provincial Hospital Affiliated to Shandong \\ University, Jinan, Shandong, People's \\ Republic of China; ${ }^{2}$ Department of \\ Physiology, Shandong University School \\ of Basic Medical Sciences, Jinan, \\ Shandong, People's Republic of China; \\ ${ }^{3}$ Department of Orthopedic, Feicheng \\ Hospital of Traditional Chinese Medicine, \\ Feicheng, Shandong, People's Republic of \\ China
}

Correspondence: Dachuan Wang Department of Spinal Surgery, Shandong Provincial Hospital Affiliated to Shandong University, Jinan, Shandong, People's

Republic of China

Tel +86 13953169759

Email wangdachuan@medmail.com.cn
Purpose: Spinal cord injury (SCI) is a relatively common, devastating traumatic condition resulting in permanent disability. In this study, the use of exosomes derived from bone mesenchymal stem cells (BMSCs-Exo) as a cell-free therapy for the treatment of SCI in rats was investigated to gain insights into their mechanisms of action.

Methods: Rats were randomly divided into three groups, Sham (treated with PBS), SCI $($ SCI injury + PBS) and SCI + Exo (SCI injury + BMSCs-Exo). Changes in the complement system between the three groups were assessed with the use of proteomics. The proteomic data were verified using reverse transcription-polymerase chain reaction (RT-PCR). In addition, the distributions of BMSCs-Exo in rats with SCI were detected by immunofluorescence. Moreover, SCI-activated NF- $\mathrm{kB}$ levels were determined using Western blot.

Results: SCI insult increased complement levels, including $\mathrm{C} 4, \mathrm{C} 5, \mathrm{C} 6, \mathrm{C} 4$ binding protein alpha and complement factor H. In contrast, the SCI + BMSCs-Exo group exhibited attenuated SCI-induced complement levels. Immunofluorescence assay results revealed that BMSCs-Exo mainly accumulated at the spinal cord injury site and were bound to microglia cells. Western blot analysis of tissue lysates showed that BMSCs-Exo treatment also inhibited SCI-activated nuclear factor kappa-B (NF- $\kappa \mathrm{B})$.

Conclusion: BMSCs-Exo play a protective role in spinal cord injury by inhibiting complement mRNA synthesis and release and by inhibiting SCI-activated NF- $\kappa$ B by binding to microglia.

Keywords: exosomes, proteomics, complement, spinal cord injury

\section{Introduction}

Spinal cord injury (SCI) represents one of the most devastating medical issues, often resulting in serious dyskinesia and even death. ${ }^{1}$ Although many therapies exist for the treatment of SCI, all have proven to be of limited efficacy. ${ }^{2}$ Accordingly, a compelling need remains for the development of novel therapeutic strategies to improve functional recovery after SCI. In experimental models of SCI, accumulating evidence indicates that mesenchymal stem cell (MSC) transplantation may represent an effective therapy for this condition. ${ }^{3,4}$ Specifically, this therapy has been shown to promote anatomical and functional recovery, as well as reducing levels of proinflammatory factors and inhibiting the nuclear factor kappa-B (NF- $\mathrm{kB}$ ) signaling pathway within these SCI models. ${ }^{5,6}$ However, direct transplantation of MSCs to target tissues remains challenging, as low survival rates, cell dedifferentiation, immune rejection, and tumor formation can all compromise the efficacy of this therapy. ${ }^{7}$ 
Exosomes are membranous lipid vesicles (diameters of 40-100 nm) that contain functional proteins, mRNA, microRNA, and substances and are involved in the process of information transfer between cells. ${ }^{8}$ They can be used to promote wound healing and skeletal muscle regeneration ${ }^{9}$ and play an important protective role in various disease models, such as liver injury, ${ }^{10}$ kidney injury, ${ }^{11}$ myocardial ischemia reperfusion ${ }^{12}$ and central nervous system injury. ${ }^{13}$ Findings from recent studies have indicated that exosomes derived from MSCs (MSCs-Exo) not only promote functional recovery after SCI by attenuating apoptosis and inflammation but also promote angiogenesis, ${ }^{2,14}$ suppress glial scar formation, attenuate lesion size, promote axonal regeneration and suppress activation of $\mathrm{A} 1$, all of which culminate in improved behavioral recovery. ${ }^{15}$ Interestingly, the results from previous studies have also demonstrated that complement inhibitors can be used as specific treatments for SCI. ${ }^{16-18}$ Furthermore, a link between extracellular vesicles and the complement system has been uncovered. ${ }^{19}$

Taken together, these findings indicate the importance of testing the hypothesis that BMSCs-Exo therapy modulates the complement system.

To test this hypothesis, Wistar rats subjected to SCI were treated with BMSCs-Exo via tail vein injection 1 $\mathrm{hr}$ following SCI insult. Changes in the complement system were assessed with use of proteomics, and SCIactivated NF- $\mathrm{KB}$ levels were determined using Western blot to reveal some of the underlying mechanisms for the beneficial effects of using BMSCs-Exo in SCI therapy.

\section{Materials And Methods}

Adult pathogen-free male Wistar rats (200-250 g) were provided by the Laboratory Animal Center of Shandong University. This study adhered to the guidelines of the Council for International Organizations of Medical Sciences for Animal Research, and experimental procedures were approved by the Animal Ethical and Welfare Committee of Shandong University.

\section{BMSCs-Exo Generation And Collection}

BMSCs were flushed from the femur of 3-4-week-old Wistar rats using a syringe. After removal, filtration and centrifugation, the supernatant was discarded, and cells were suspended in culture medium and adjusted to a density of $2.0 \times 10^{5}$ cells $/ \mathrm{mL}$ after cultivation in $25 \mathrm{~cm}^{2}$ culture flasks to generate the original cells. Medium was replaced after 24 hrs and every 2-3 days thereafter until cells had been expanded to 5 passages. ${ }^{12}$ After removing the culture medium and washing three times with PBS, DMEM/F12 was added with exosome-free serum. After $24 \mathrm{hrs}$, cultures were collected into $50 \mathrm{~mL}$ centrifugation tubes. BMSCs-Exo were isolated from the supernatant of MSCs by a multistep process of centrifugation using the QEV kit (iZon Science, Cambridge, MA) according to the manufacturer's instructions. BMSCs-Exo were collected into $1.5 \mathrm{~mL}$ EP tubes and stored at $-80^{\circ} \mathrm{C}$ for later use. Total protein concentrations of BMSCs-Exo samples were measured using the BCA protein assay kit CW0014S (CWBIO; Haimen, Jiangsu, China). BMSCs-Exo morphology was assessed using transmission electron microscopy as described by Kosanovic. ${ }^{20}$ Western blot was used to detect expression levels of CD9 and TSG101 markers.

\section{SCl Model And Treatments}

All rats were permitted free access to water and food and were housed individually in cages in a temperature and light cycle controlled environment.

A spinal cord hemisection injury model was used in this study. Rats were anesthetized using 4\% isoflurane and anesthesia was maintained with $2 \%$ isoflurane. The T10 spinous process was located, and the skin and subcutaneous tissue over the T10-12 area was incised, bilateral perispinous muscles were separated, and the T10 spinous process and vertebral plate exposed. A small needle holder was used to sever the right vertebral plate at the medial surface of the right articulation process. The spinous process was then removed to expose a small region of the left spinal cord. After identifying half of the right and median veins of the spinal cord, the median vein was used as a boundary where, slightly to the left of the center, the right semicircular spinal cord was severed horizontally using an iris knife. To be considered an effective SCI model, the following inclusion criteria were required: 1) in the postoperative quiet state, no right hind limb joint movement or slight left hind limb join movement were observed, 2) the tail of the rats showed a left curl and 3) the Basso, Beattie and Bresnahan locomotor rating scale (BBB score) was less than or equal to $1 .^{21}$ The sham group underwent only laminectomy.

Experimental animals (73 surgeries) were randomly divided into three groups (Table 1): 1) Sham (treated with PBS), 2) SCI (SCI injury + PBS) and 3) SCI + Exo (SCI injury + BMSCs-Exo). BMSCs-Exo $(500 \mu \mathrm{L})$ was injected into the tail vein $(100 \mu \mathrm{g}$ total protein of exosome 
Table I Sizes Of Each Group/experiment In This Study

\begin{tabular}{|l|l|l|}
\hline Experiment & Size Of Each Group & $\mathbf{N}$ \\
\hline Behavioral tests & $\begin{array}{l}\text { Sham }(\mathrm{N}=5), \mathrm{SCl}(\mathrm{N}=5), \mathrm{SCl}+ \\
\text { Exo }(\mathrm{N}=5)\end{array}$ & 15 \\
\hline Proteomics & $\begin{array}{l}\text { Sham }(\mathrm{N}=6), \mathrm{SCl}(\mathrm{N}=6), \mathrm{SCl}+ \\
\text { Exo }(\mathrm{N}=6)\end{array}$ & 18 \\
\hline $\begin{array}{l}\text { Extraction and quantitative } \\
\text { analysis of mRNA }\end{array}$ & $\begin{array}{l}\text { Sham }(\mathrm{N}=4), \mathrm{SCl}(\mathrm{N}=4), \mathrm{SCl}+ \\
\text { Exo }(\mathrm{N}=4)\end{array}$ & 12 \\
\hline $\begin{array}{l}\text { Immunohistochemistry } \\
\text { staining }\end{array}$ & $\begin{array}{l}\text { Sham(N=4), SCl (N=4), SCl + } \\
\text { Exo }(\mathrm{N}=4)\end{array}$ & 12 \\
\hline Immunofluorescence staining & $\begin{array}{l}\text { SCl + Exo (PKH labeled) } \\
(\mathrm{N}=4)\end{array}$ & 4 \\
\hline Western blot analysis & $\begin{array}{l}\text { Sham (N=4), SCl (N=4), SCl + } \\
\text { Exo }(\mathrm{N}=4)\end{array}$ & 12 \\
\hline Total & $\begin{array}{l}\text { Sham (N=23), SCl (N=23), } \\
\text { SCl + Exo (N=27) }\end{array}$ & 73 \\
\hline
\end{tabular}

precipitate in $500 \mu \mathrm{L}$ PBS, injection speed $=500 \mu \mathrm{L} / \mathrm{min}$ ) of the SCI + Exo group $1 \mathrm{hr}$ after SCI. For Sham and SCI groups, $500 \mu \mathrm{L}$ PBS $(500 \mu \mathrm{L} / \mathrm{min})$ were injected into the tail vein as described for the SCI + Exo group $1 \mathrm{hr}$ after Sham/SCI treatment.

\section{Behavioral Tests And Morphological Assessment}

BBB scores were used to evaluate functional recovery of hind limb locomotion. ${ }^{21}$ Evaluations were conducted 1, 3, 7, 14, 21 and 28 days postinjury by two well-trained observers who were blinded to experimental animal grouping. For these tests, rats were allowed to move freely in an open field for 4 min, and average locomotor scores were calculated and recorded.

\section{Incline Plate Test}

A $6 \mathrm{~mm}$ thick rubber pad was placed on the surface of an inclined plate, and rats were placed in a body axis direction perpendicular to the longitudinal axis of the inclined plate. The angle of inclination was gradually increased, and rats were required to stay on the inclined plate for at least $5 \mathrm{~s}$ to record the maximum angle reached. Each rat was measured three times during each session, and the average value was recorded. The incline plate test was recorded 1, 3, 7, 14, 21, and 28 days post-SCI in all three groups.

\section{Tandem Mass Tag (TMT) Quantitative Proteomics}

TMT technology involves isotope labeling of relative and absolute quantitative reagent technology as developed by the Thermo SCIENTIFIC Company. ${ }^{22}$ Three groups of samples were analyzed using this technology.

Seventy-two hours after Sham/SCI procedures, a certain number of rats were euthanized, spinal cords were immediately collected, and total protein was extracted from each tissue. Protein concentrations were determined using a Bradford assay. After trypsin digestion, peptides were desalted with a $\mathrm{C} 18$ cartridge to remove high concentrations of urea. Desalted peptides were then dried by vacuum centrifugation. After being labeled with TMT, peptides were fractionated using a $\mathrm{C} 18$ column on a Rigol L3000 HPLC. Samples were then dried using a vacuum and reconstituted in $0.1 \%(\mathrm{v} / \mathrm{v})$ formic acid (FA) in water for subsequent LC-MS/MS analysis. This analysis was performed by Novogene Bioinformatics Technology Co. Ltd.

\section{Proteomics Data Normalization And Analysis}

Raw files obtained from LC-MS/MS were searched using corresponding databases, and protein identification was performed based on the results of this database search. Mass spectrometry data were searched using the UniProt database (rattus_norvegicus_uniprot_2018.07.02.fasta) with the use of Proteome Discoverer 2.2 software. GeneOntology (GO) and Kyoto Encyclopedia of Genes and Genomes (KEGG) pathway were used to identify the potential function of all proteins.

Analysis of protein differences initially selects sample pairs that require comparisons and then determines the ratio of the mean value of all biological repeat quantitative values of each protein in the comparison sample pairs as a fold change (FC). To determine the statistical significance of these differences, $t$-tests were conducted on the relative quantitative value of each protein in the two samples for comparison, and the corresponding $p$-value was calculated as a significance index. When $p<0.05$ and $\mathrm{FC} \geq 1.2$, the protein expression level was upregulated, whereas $\mathrm{p}<0.05$ and $\mathrm{FC} \leq 0.83$ indicated that the protein expression level was downregulated. Up- and downregulated proteins were then screened according to these criteria. 


\section{Extraction And Quantitative Analysis Of mRNA}

Spinal cord injury samples were harvested at $72 \mathrm{hrs}$ post-injury. Total RNA was extracted from samples using TRIzol reagent (CWBIO Jiangsu, China). According to manufacturer's instructions, RNA reverse transcription to complementary DNA (cDNA) was accomplishing using specific primers. All primers (Table 2) were designed and synthesized by BioSune Biotechnology (Shanghai). PCR results were normalized to $\beta$-actin expression. Electrophoresis was used to separate reaction products on $1.2 \%$ agarose/TAE gels, and reaction products were then run for 30-40 min at 90 V. The GelDocXR System was used to capture images. Image-Pro Plus 6.0 software was used to determine band intensities, and each value was normalized to $\beta$-actin.

\section{Immunohistochemistry Staining}

At $72 \mathrm{hrs}$ post-injury, the SCI site was extracted and then fixed in $4 \%$ paraformaldehyde for $24 \mathrm{hrs,} \mathrm{embedded} \mathrm{in}$ paraffin and sliced into $5 \mu \mathrm{m}$ thick sections. Slides were dewaxed, incubated overnight at $4^{\circ} \mathrm{C}$ with primary antibody C1q (ab71089, Abcam), then blocked with secondary antibody and visualized with DAB. Nuclei were counterstained with hematoxylin. Sections were observed using a histologic microscope (OLYMPUS-BX51) and counting was performed in a blinded manner.

\section{Immunofluorescence Staining}

The green fluorescent dye PKH67 (Sigma-Aldrich, Olney, USA) was used to label MSCs-Exo according to manufacturer's instructions. PKH67-labeled exosomes were injected through the tail vein $1 \mathrm{hr}$ after SCI. At $72 \mathrm{hrs}$ ' post-injury, the spinal cord injury site was extracted and then fixed in $4 \%$ paraformaldehyde for $24 \mathrm{hrs}$ with $30 \%$ sucrose for dehydration. In addition, the liver, kidney and spleen were also extracted and then fixed at day 1, day 3 and day 7 after SCI. The primary antibody Iba-1 (1:100 10904-1-AP, Proteintech) was used. After incubation with the primary antibody, samples were washed and blocked with the appropriate secondary antibody. Sections were observed by confocal fluorescence microscopy (OLYMPUS-BX51) and counting was performed in a blinded manner.

\section{Western Blot Analysis}

Protein lysates were extracted from tissues of injured spinal cord tissue $72 \mathrm{hrs}$ post-injury. Protein concentration was determined using the BCA protein assay kit (CW0014S, CWBIO; Haimen, Jiangsu, China). Concentrations of 20-40 $\mu \mathrm{g}$ total protein were separated on polyacrylamide gels, transferred to polyvinylidene difluoride membranes and incubated overnight at $4{ }^{\circ} \mathrm{C}$ with the following primary antibodies: NF- $\kappa B$ (p65) (10745-1-AP, Proteintech), p-NF$\kappa B$ (Ser536) (\#3033, Cell Signaling), IкB $\alpha$ (10268-1-AP, Proteintech Group), p-IкB $\alpha$ (\#2859, Cell Signaling), and $\beta$ actin (TA-09, Zhongshan Golden Bridge Biotechnology, Beijing, China). Membranes were incubated with secondary antibodies, and signals were visualized using enhanced chemiluminescence.

\section{Statistical Analysis}

All experiments were repeated three times. And the size of most of the groups is $n=4-6$; therefore, non-parametric tests (Kruskal-Wallis test followed by post hoc \{DunnBonferroni test $\}$ ) were used to compare different groups. Data are shown as the median (min-max). Statistical analysis was conducted using SPSS 25.0 (IBM Corp., Armonk, NY, USA). $P$-values $<0.05$ were considered statistically significant.

Table 2 Primer Sequences Used In The Study

\begin{tabular}{|l|l|l|}
\hline Gene & Forward $\left(\mathbf{5}^{\prime} \boldsymbol{\rightarrow} \mathbf{3}^{\prime} \mathbf{)}\right.$ & Reverse $\mathbf{( 5}^{\prime} \rightarrow \mathbf{3}^{\prime} \mathbf{)}$ \\
\hline Actin & CTCTGTGTGGATTGGTGGCT & CGCAGCTCAGTAACAGTCCG \\
$\mathrm{Mbll}$ & CTTCCTAGGCATCACGGACG & TCCAGTTGCTGTAGGTGAGC \\
$\mathrm{Cl} q$ & GGGACGAGGTGTGGATTGAG & GGCCGAGGGAAAAATGAGGA \\
$\mathrm{C} 4 \mathrm{~b}$ & ATTGGTGACTCCCCCGAGA & GCCTCTTTGAATGACCGGA \\
$\mathrm{Cfh}$ & AAGGCACTCAGGCAACCTAC & CCAACTGCCAGCCTAAAGGA \\
$\mathrm{C5}$ & ATCAGGGCCTTCAACGAGTG & GCCGATGCCTTGAATTTCCC \\
$\mathrm{C6}$ & CAGATGCTTACCAGACAGAACCT & TGGGACAGGTCAGCTCAATG \\
$\mathrm{Cfp}$ & AGGTGCAAAGGCCTACTTGG & CTGGAAGGCATAGGCAGTGT \\
$\mathrm{C3}$ & TTTCGAAGTCCTGGTGGAGC & TCCCCTGAACCATCCTCGAT \\
\hline
\end{tabular}




\section{Results}

\section{Characterization Of BMSCs-Exo}

Transmission electron microscopic observation of BMSCsExo revealed the presence of typical cup-shape spherical vesicles. The size distribution profile indicated a homogeneous population ranging from 20 to $130 \mathrm{~nm}$ (Figure 1A). Western blot was used to examine the presence of the exosome markers CD9 and TSG101 (Figure 1B).

\section{BMSCs-Exo Improved Functional Recovery After SCl}

Gradual increases in BBB scores in both SCI and SCI + Exo groups demonstrated that functional recovery occurred over the post-paraplegia surgery evaluation period (Figure 1C). However, while all SCI rats (SCI and SCI + Exo groups) showed similar paraplegic hind limb symptoms with BBB scores of $\leq 1$ on day 1 , the scores in the $\mathrm{SCI}+$ Exo group were significantly higher than those of rats in the SCI group on days $7(p<0.05), 14(p<0.01), 21$ and $28(p<0.05)$ after SCI. The results of incline plate test were consistent with the BBB score (Figure S1). All these findings demonstrated that the $\mathrm{SCI}+$ Exo group exhibited much better recovery compared with the SCI group, indicating that BMSCs-Exo treatment significantly improved motor function in rats with SCI.

\section{Differential Proteomic Analysis Of The Complement System}

KEGG analysis revealed that significant differences were present in complement protein enrichment among
Sham, SCI and SCI + Exo groups (Figure 2). Twentyfour upregulated proteins were observed between SCI and Sham groups $(p<0.05, \mathrm{FC} \geq 1.2)$ (Table 3). Compared to the Sham group, the complement system was upregulated and activated in the SCI group. These upregulated proteins included complement factor $\mathrm{H}$ (Cfh), complement factor properdin (Cfp), complement factor B (Cfb), complement C5 (C5), complement component C6 (C6), Cfh protein, mannose-binding protein A (Mbl), complement C3 (C3), complement C4 (C4), complement component C9 (C9), complement C8 alpha chain (C8a), C4b-binding protein alpha chain (C4bpa), complement factor I (Cfi), plasma protease C1 inhibitor (Serping1), complement component $\mathrm{C} 8$ beta chain (C8b), complement C7 (C7), complement C2 (C2), complement $\mathrm{C} 1 \mathrm{q}$ subcomponent subunit B (C1qb), C1r protein $(\mathrm{C} 1 \mathrm{r})$, complement $\mathrm{C} 8$ gamma chain $(\mathrm{C} 8 \mathrm{~g})$, Complement $\mathrm{C} 1 \mathrm{q}$ subcomponent subunit $\mathrm{C}(\mathrm{C} 1 \mathrm{qc})$, carboxypeptidase B2 (Cpb2), complement $\mathrm{C} 1 \mathrm{~s}$ subcomponent $(\mathrm{C} 1 \mathrm{~s})$ and mannan-binding lectin serine protease 1 (Masp1).

Seven downregulated proteins were identified in the $\mathrm{SCI}+$ Exo group compared to the SCI group $(p<0.05$, FC $\leq 0.83$ ) (Table 4). These downregulated proteins included Cfh, C5, C6, Cfh protein, Cfp, C4bpb and Mbl. In contrast to the SCI alone group, rats in the SCI + Exo treatment group demonstrated the capacity to downregulate some components of the complement system, resulting in complement system inhibition.
$\mathbf{A}$

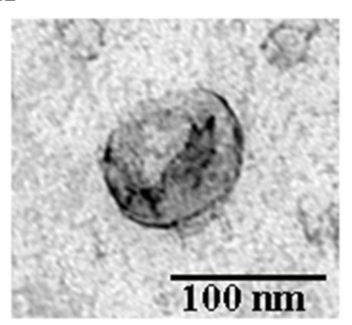

B

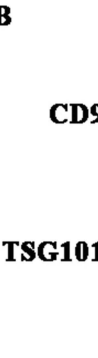

Calnexin

p-actin
BMSCs-Exo
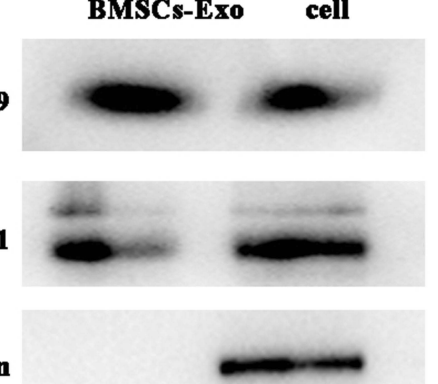

C

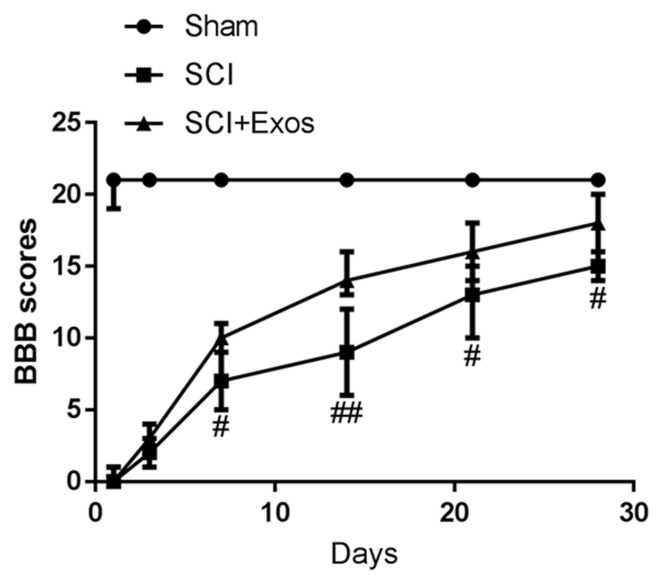

Figure I Morphology of BMSCs-Exo as assessed by transmission electron microscopy and BBB scores.

Notes: (A) Presence of typical cup-shape spherical BMSCs-Exo. The size distribution profile indicated a homogeneous population with sizes ranging from 20-I30 nm. Scale bar $=100 \mathrm{~nm}$. (B) Western blot analysis of specific surface markers of BMSCs-Exo, TSGI0I and CD9. (C) BMSCs-Exo improve functional recovery after SCI. Open-field $\mathrm{BBB}$ scores recorded at I, 3, 7, I4, 2I, and 28 days post-SCI in the three groups. Functional recovery improved gradually over time throughout the evaluation period in both $\mathrm{SCl}$ and $\mathrm{SCl}+$ Exo groups. Compared to the $\mathrm{SCl}$ group, rats of the $\mathrm{SCl}+$ Exo group showed significantly greater improvements in functional activity over the observation period. All data are presented as the median (min-max). \#p<0.05, \#\#<0.0I, $\mathrm{SCl}+$ Exo versus $\mathrm{SCl}$. 


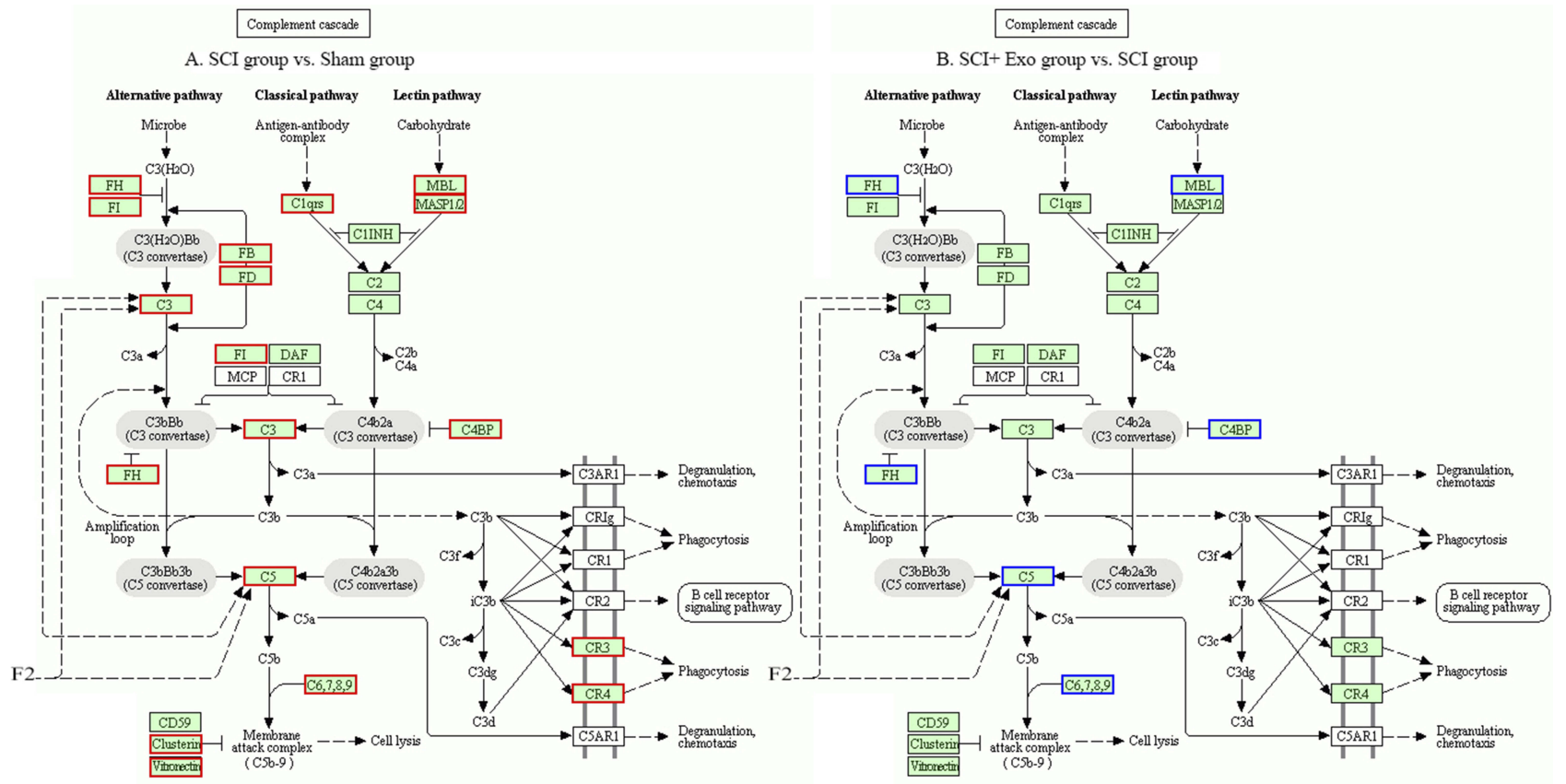

Figure 2 Complement cascade Kyoto Encyclopedia of Genes and Genomes (KEGG) pathway map.

Notes: Differentially expressed proteins are circled in the KEGG diagram. (A) SCl versus Sham; (B) SCl + Exo versus SCl. Green background color frame indicates total proteins. Blue frame indicates differentially expressed downregulated proteins $(p<0.05, \mathrm{FC} \leq 0.83)$. Red frame indicates differentially expressed upregulated proteins $(p<0.05$, $\mathrm{FC} \geq \mathrm{I}$.2) (KEGG map046I0).

\section{BMSCs-Exo Attenuated SCl-Induced Increases In Complement mRNA}

Expression of complement mRNA was examined using RTPCR, and results from this assay verified the presence of corresponding genes from 8 proteins in the complement system, including Mbl, C1q, C4b, Cfh, C5, C6, Cfp and C3 (Figure 3A). Compared with the Sham group, complement mRNA in the SCI group was significantly increased for C1q, C3, C4b, C6, C5 and Mbl $(p<0.001)$, Cfh $(p<0.01)$ and Cfp $(p<0.05)$. After treatment with BMSCs-Exo, the levels of complement mRNA in the SCI + Exo group were significantly decreased compared to the SCI group for $\mathrm{Clq}$ and $\mathrm{Cfh}$ $(p<0.01)$ and C3, C4b, C6, C5, Mbl and Cfp $(p<0.05)$.

\section{Effect Of BMSCs-Exo On Clq Expression As Determined By Immunohistochemistry Of Injured Tissue} As C1q plays a key role in the complement system in SCI, we investigated SCI-induced changes in $\mathrm{Clq}$ expression in response to BMSCs-Exo treatment $72 \mathrm{hrs}$ after SCI (Figure 3B). Immunohistochemistry analysis revealed very few C1q-positive cells in spinal tissue samples in the Sham group, whereas C1q-positive cells were numerous in the SCI group. BMSCs-Exo treatment significantly attenuated $\mathrm{C} 1 \mathrm{q}$ expression within SCI + Exo group compared to the SCI group.

\section{Immunofluorescence Staining}

We firstly explored the distribution of exosomes in different tissues including liver, kidney and spleen at day 1, day 3 and day 7 after SCI. And the results (Figure S2) showed that the fluorescence intensity was the strongest on the first day, and the fluorescence intensity gradually decreased at Day 3 and Day 7. The fluorescence approximately disappeared at Day 7, which indicated that the exosomes existed in the tissue for about 7 days.

Immunofluorescence results revealed that Iba-1 was profusely expressed near the injury site in all SCI rats (Figure 4). Large numbers of labeled BMSCs-Exo were found in microglial cells, with most of these BMSCs-Exo accumulating within small clusters in the cytoplasm of microglia.

\section{BMSCs-Exo Inhibits SCl-Induced NF-kB Activation}

The results of Western blot analysis revealed that phosphory-

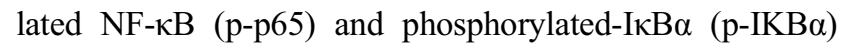
levels were significantly upregulated in response to $\mathrm{SCI}$ compared to the Sham group $(p<0.01)$ (Figure 5). After 
Table 3 Upregulated Complement Proteins (SCI Group Vs Sham Group) Identified By Proteomics ( $p<0.05$ And FC $\geq 1.2)$

\begin{tabular}{|c|c|c|c|c|}
\hline Accession & Description & Gene & SCI vs Sham FC & SCl vs Sham $P$-value \\
\hline G3V9R2 & Complement factor $\mathrm{H}$ & $C f h$ & 2.10 & $9.01 E-04$ \\
\hline A0A096P6L9 & Complement C5 & $C 5$ & 1.73 & I.79E-05 \\
\hline Q8IIM5 & Complement component $\mathrm{C} 6$ & C6 & 1.61 & $6.68 \mathrm{E}-03$ \\
\hline Q5FVP9 & Cfh protein & & 2.19 & 8.79E-04 \\
\hline BOBNN4 & Complement factor properdin & $C f p$ & 2.29 & 8.13E-03 \\
\hline PI9999 & Mannose-binding protein A & Mbll & 1.47 & I.08E-03 \\
\hline MORBFI & Complement $\mathrm{C} 3$ & C3 & 2.17 & $6.18 \mathrm{E}-05$ \\
\hline P08649 & Complement C4 & C4 & 1.62 & $3.56 \mathrm{E}-06$ \\
\hline G3V6I5 & Complement factor $B$, isoform CRA_b & $C f b$ & 2.28 & I.72E-04 \\
\hline F7F389 & Complement component C9 & C9 & 2.04 & $1.92 \mathrm{E}-04$ \\
\hline D3ZWD6 & Complement $\mathrm{C} 8$ alpha chain & $\mathrm{C} 8 \mathrm{a}$ & 1.66 & 7.57E-04 \\
\hline Q5M89I & C4b-binding protein alpha chain & C4bpa & 2.00 & $3.65 \mathrm{E}-03$ \\
\hline A0A0G2KI35 & Complement factor I & $C f i$ & 1.58 & $3.89 \mathrm{E}-02$ \\
\hline Q6P734 & Plasma protease $\mathrm{Cl}$ inhibitor & Serping I & 1.64 & 4.27E-04 \\
\hline P553।4 & Complement component $\mathrm{C} 8$ beta chain & $C 8 b$ & 1.80 & $6.7 I E-04$ \\
\hline AOA0G2K7X7 & Complement C7 & $C 7$ & 1.62 & $1.03 \mathrm{E}-03$ \\
\hline Q6MG73 & Complement C2 & $C 2$ & 1.45 & $1.58 \mathrm{E}-02$ \\
\hline G3V7N9 & Complement $\mathrm{Clq}$ subcomponent subunit $\mathrm{B}$ & $C l q b$ & 1.66 & 8.95E-04 \\
\hline B5DEH7 & $\mathrm{CIr}$ protein & $\mathrm{Cl} r$ & 1.28 & $3.50 \mathrm{E}-02$ \\
\hline D3ZPI8 & Complement C8 gamma chain & $\mathrm{C} 8 \mathrm{~g}$ & 1.33 & I.60E-02 \\
\hline $\mathrm{AOAOH} 2 \mathrm{UHKI}$ & Complement $\mathrm{Clq}$ subcomponent subunit $\mathrm{C}$ & $\mathrm{Clqc}$ & 2.20 & 3.47E-04 \\
\hline Q9EQV9 & Carboxypeptidase B2 & Cpb2 & 1.93 & 5.47E-04 \\
\hline G3V7L3 & Complement $\mathrm{Cl}$ s subcomponent & $\mathrm{Cls}$ & 1.34 & $2.58 \mathrm{E}-03$ \\
\hline AOAOH 2 UHAI & Mannan-binding lectin serine protease I & Maspl & 1.42 & $7.86 \mathrm{E}-03$ \\
\hline
\end{tabular}

Table 4 Downregulated Complement Proteins (SCl+exo Group Vs SCl Group) Identified By Proteomics ( $p<0.05$ And FC $\leq 0.83$ )

\begin{tabular}{|l|l|l|l|l|}
\hline Accession & Description & Gene & SCI+Exo vs SCI FC & SCI+Exo vs SCI P-value \\
\hline G3V9R2 & Complement factor H & Cfh & 0.76 & $3.09 \mathrm{E}-02$ \\
A0A096P6L9 & Complement C5 & C5 & 0.75 & $2.52 \mathrm{E}-02$ \\
Q8IIM5 & Complement component C6 & C6 & 0.76 & $2.74 \mathrm{E}-02$ \\
Q5FVP9 & Cfh protein & & 0.74 & 4.1 E-02 \\
B0BNN4 & Complement factor properdin & Cfp & 0.72 & $2.40 \mathrm{E}-02$ \\
A0A5C5 & C4b-binding protein beta chain & C4bpb & 0.75 & 0.049 \\
PI9999 & Mannose-binding protein A & Mbll & 0.78 & $6.85 \mathrm{E}-04$ \\
\hline
\end{tabular}

BMSCs-Exo treatment, p-p65 and p-IкB $\alpha$ levels were significantly downregulated compared to the SCI group (p-p65, $p<0.05$; $\mathrm{p}-\mathrm{IKB} \alpha, p<0.01)$.

\section{Discussion}

In this study, we demonstrated that BMSCs-Exo play a protective role in spinal cord injury by inhibiting complement mRNA synthesis and release and by inhibiting SCIinduced activation of $\mathrm{NF}-\kappa \mathrm{B}$. Moreover, the results from immunofluorescence revealed that BMSCs-Exo primarily accumulated at the site of spinal cord injury and were bound to microglial cells.

\section{Complement System Function After SCl}

The complement system represents an important component of the immune system and affects amplification of this system in vivo. ${ }^{23}$ Normally, activation of the complement system is strictly regulated. However, overactivation of complement, as can result from changes in the internal and/or external environment of some organisms, can lead to complement dysfunction. Such an effect can then produce damage to multiple tissues and organs. ${ }^{24}$

SCI can lead to complex, multiphasic inflammatory responses during the early phases of injury, ${ }^{25}$ which can then damage spinal cord cells. The results from previous 

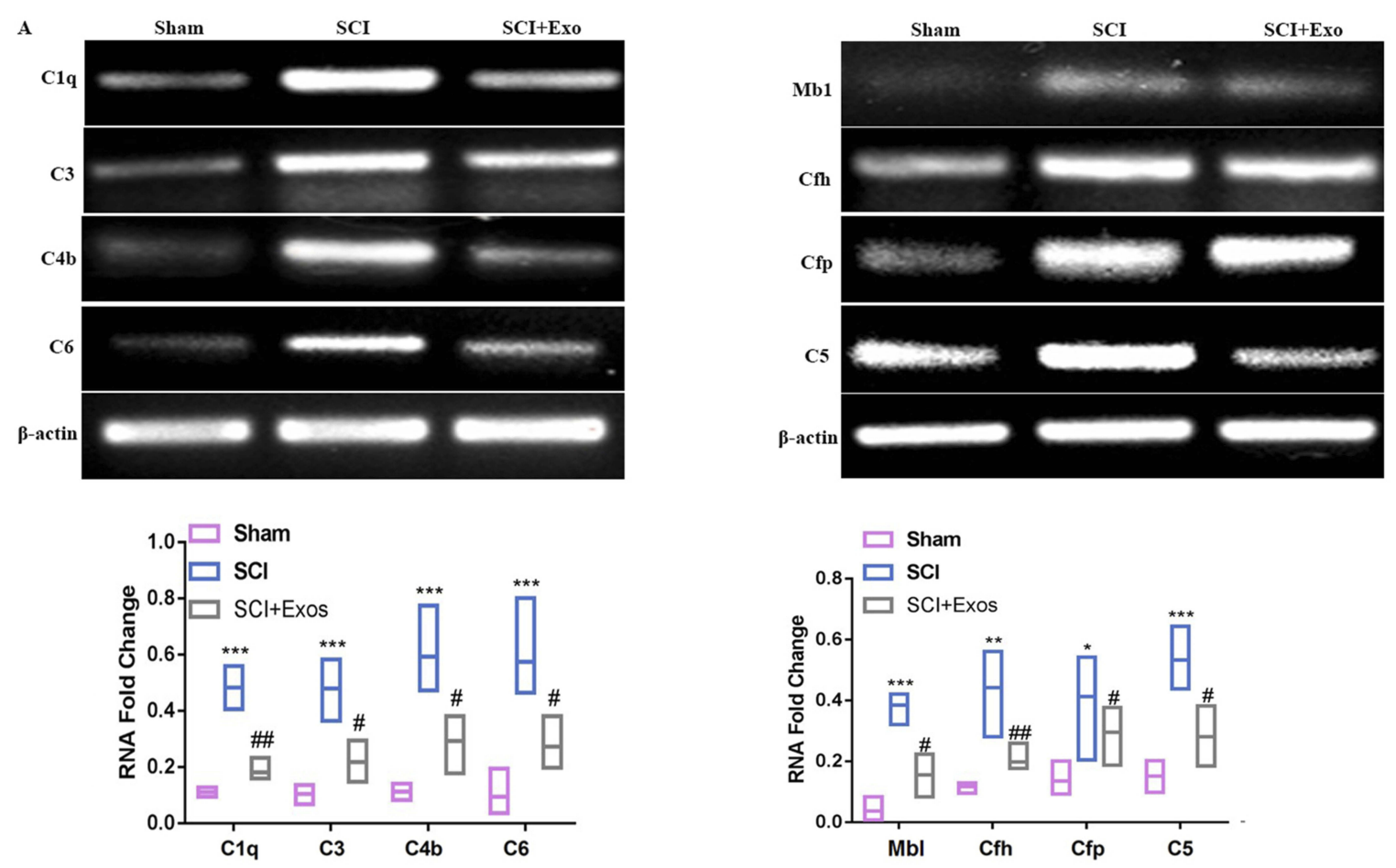

B
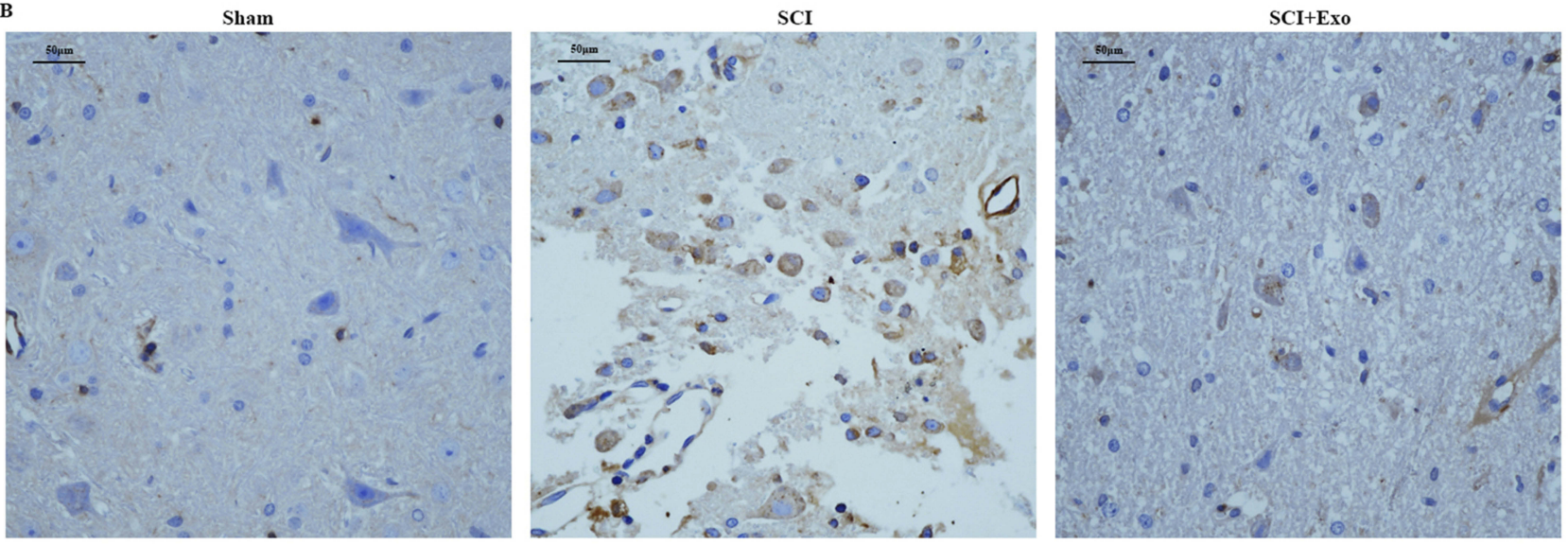

Figure 3 BMSCs-Exo attenuate $\mathrm{SCl}$-increased complement.

Notes: (A) Expression of complement mRNA was examined using RT-PCR and included Mbl, Clqa, C4b, Cfh, C5, C6, Cfp and C3. Compared to the Sham group, complement mRNA in the SCl group was significantly increased. After treatment with BMSCs-Exo, levels of complement mRNA in the SCl + Exo group were significantly

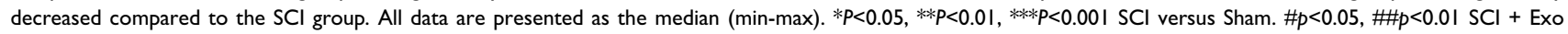
versus $\mathrm{SCl}$. (B) Effect of BMSCs-Exo on expression of $\mathrm{Clq}$ immunohistochemistry in injured tissue. Scale bar $=50 \mu \mathrm{m}$.

experiments have shown that treatments that reduce such inflammation have beneficial effects in moderating SCI. ${ }^{17}$ Interestingly, inflammation activates the complement system; however, this activation is not sufficient to inhibit the inflammatory response. In fact, this activation may even aggravate SCI-induced inflammatory responses, thus enhancing the harmful effects of SCI. ${ }^{26}$

Complement activation represents one of the critical initial components of neuro-inflammatory responses associated with SCI. It has been reported that complement is elevated within 1 day post-SCI in rodents, ${ }^{27}$ and this phenomenon also been observed in human studies of SCI. ${ }^{28}$ Results from previous experiments have shown that complement proteins (clusterin, C1q, MAC, Cfh, $\mathrm{CFB}, \mathrm{C} 3, \mathrm{C} 4, \mathrm{C} 9)$ are significantly increased in rat models of SCI. ${ }^{29}$ Moreover, complement components (Cfh, $\mathrm{Cfb}$, $\mathrm{C} 1 \mathrm{q}, \mathrm{C} 4, \mathrm{C} 5 \mathrm{~b}, \mathrm{C} 6-\mathrm{C} 9)$ are deposited in the neurological tissue of rats post-SCI. ${ }^{27}$ In addition to complement protein synthesis by hepatocytes, it was reported that neutrophils, macrophages and $\mathrm{T}$ lymphocytes can produce 

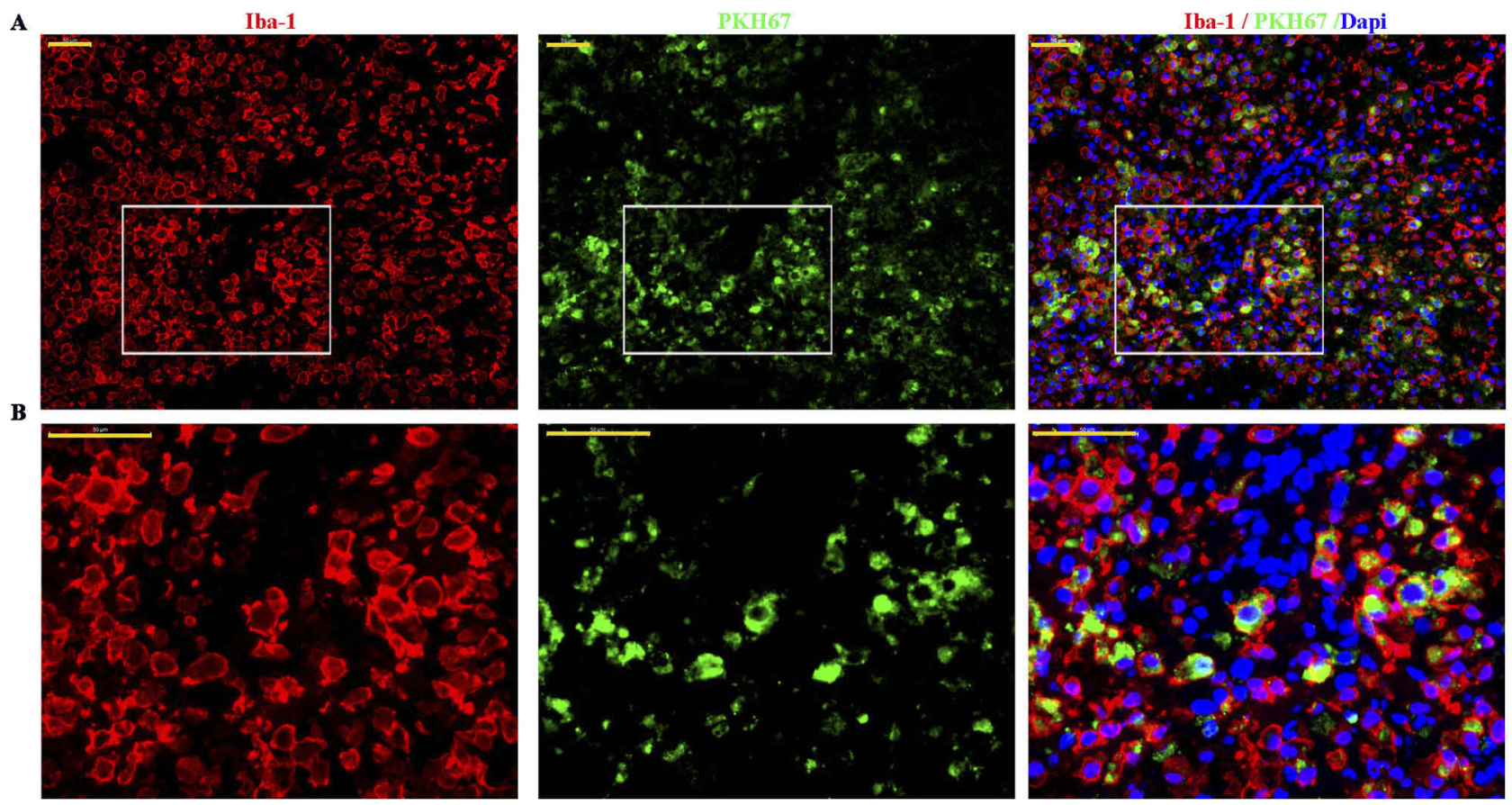

Figure 4 BMSCs-Exo selectively target microglia/macrophages in damaged spinal cord.

Notes: (A) Representative images of double Immunofluorescence staining of microglia/macrophages (red, Ibal) and PKH67-labeled MSCs-Exo (green) within the damaged spinal cord. (B) Partially enlarged images of microglia (red, lbal) and PKH67-labeled MSCs-Exo (green). Scale bar $=50 \mu \mathrm{m}$.

complement proteins in response to SCI, leading to increased complement activation. ${ }^{30}$

Our current results revealed that 24 complement proteins, including Cfh, Cfp, Cfb, C5, C6, Mbl, C3, C4, C9 and $\mathrm{C} 4 \mathrm{bp}$, were upregulated in response to SCI. These results are consistent with previous findings. ${ }^{27,29}$ As C3 and $\mathrm{C} 1 \mathrm{q}$ are key components of the complement system, we focused on these factors using RT-PCR. Our results differ from those obtained with proteomics, which we hypothesized may be related to post-transcriptional modification of these proteins.

\section{The Complement System As A Target For Treatment Of SCl}

Accumulating evidence indicates that inhibition or deficiency of $\mathrm{C} 3 / \mathrm{C} 5$ protects against inflammation, reduces injury and improves histological and functional locomotor outcomes after SCI. ${ }^{17,18,24,31} \mathrm{C} 1 \mathrm{q}$ deficiency has been shown to improve neural regeneration and functional recovery after $\mathrm{SCI},{ }^{32}$ while a deficiency of $\mathrm{Cfb}$ reduces demyelination and inflammation, improving functional recovery from SCI in mice. ${ }^{24}$ Moreover, intravenous injection of CR2-Crry (mouse complement inhibition) improves functional recovery from $\mathrm{SCI}$ and protects against neurological tissue damage resulting from $\mathrm{SCI}^{24,33}$ In the study, we demonstrate that complement components were markedly downregulated by BMSCs-Exo treatment in SCI rats compared the SCI group. Taken together, these data uncover two significant novel findings. First, the beneficial effects of BMSCs-Exo treatment in SCI rats involve, in part, their capacity to downregulate complement components. Second, the complement system may represent an important new target for improving post-traumatic recovery from SCI.

\section{Effects Of BMSCs-Exo On The Complement System Following SCI}

There is considerable evidence indicating the importance of MSCs-Exo on various bodily functions. For example, $\mathrm{Toh}^{34}$ reported that MSCs-Exo carry large functional proteins and play an important role in modulating immunomodulatory activities, while Eirin $^{35}$ reported that porcine adipose tissue-derived MSCs-Exo play an important role in inflammation or complement activation, as determined by proteomic profiling. Moreover, there is accumulating evidence that complex interactions exist between exosomes and complement systems, which play an important role in local and systemic inflammation; ${ }^{19,36}$ and, increased numbers of interactions were found in the circulation of patients during inflammatory conditions with highly activated complement. ${ }^{37,38}$ Results from some 

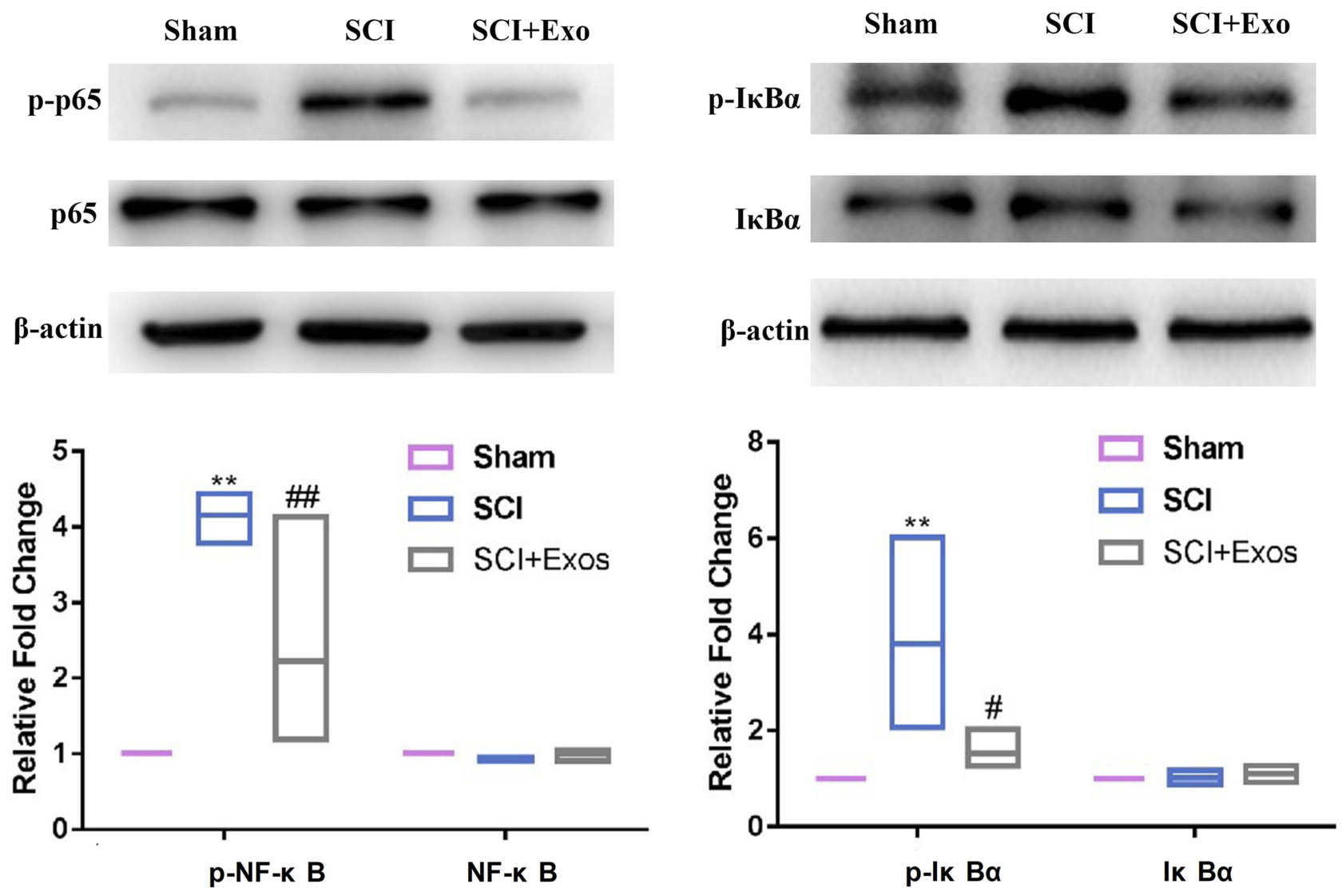

Figure 5 BMSCs-Exo inhibit SCl-induced NF-kB activation.

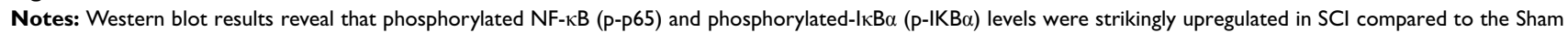
group. After BMSCs-Exo treatment, $\mathrm{p}-\mathrm{p} 65$ and $\mathrm{p}-\mathrm{IKB} \alpha$ levels were significantly downregulated compared to the $\mathrm{SCl}$ group. All data are presented as the median (min-max). $* * P<0.0 \mathrm{I} \mathrm{SCl}$ versus Sham. \#p<0.05, \#p<0.0I SCl + Exo versus SCl.

current studies indicate that the surface of exosomes contains key complement factors and complement regulators that affect inflammation and disease progression. ${ }^{37,39-41}$

In our present study, we identified 7 downregulated complement proteins in the SCI + Exo group using proteomic profiling. These proteins included $\mathrm{Cfh}, \mathrm{C} 5, \mathrm{C} 6, \mathrm{Cfh}$ protein, $\mathrm{Cfp}, \mathrm{C} 4 \mathrm{bpb}$ and $\mathrm{Mbl}$. Moreover, the results from RT-PCR and immunohistochemistry assays revealed that BMSCs-Exo treatment also inhibited activation of $\mathrm{Clq}$ in response to $\mathrm{SCI}$, which is likely related to the functional recovery of locomotor responses observed in these rats.

\section{BMSCs-Exo Selectively Target Microglia Cells In Damaged Spinal Cord}

Microglia cells are important immune effector cells in the central nervous system (CNS) and play an extremely important role in physiological processes of the CNS. Specifically, they mediate endogenous immune responses to CNS damage and disease and exert neuroprotective effects. ${ }^{42,43}$ In the treatment of encephalopathy, exosomes primarily target microglia and neuronal cells. ${ }^{44,45}$ In this study, immunofluorescence results of PKH67-labeled BMSCs-Exo indicated their presence within the spinal cord injury area and were primarily localized to microglial cells. These findings suggest that BMSCs-Exo may have several cellular targets in SCI, with microglial cells being a major target in SCI. Thus, microglia cells may play crucial roles in post-injury functional recovery after SCI.

\section{BMSCs-Exo Inhibited SCl-Induced NF-kB Activation}

It has been reported that complement $(\mathrm{C} 3 / \mathrm{C} 5)$ is related to the NF- $\kappa \mathrm{B}$ signaling pathway. ${ }^{46-48}$ Huang et al observed that systemic administration of exosomes after SCI can reduce inflammation. ${ }^{2}$ After $\mathrm{SCI}$, the $\mathrm{NF}-\mathrm{\kappa B}$ signaling pathway is activated, and secondary inflammatory responses are regulated by $\mathrm{NF}-\kappa \mathrm{B}$, while inhibition of $\mathrm{NF}-\kappa \mathrm{B}$ promotes functional recovery after $\mathrm{SCI}^{49}$ In our

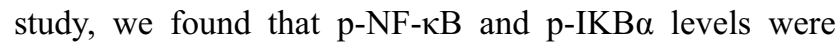
markedly downregulated by BMSCs-Exo treatment 
compared to the SCI alone group, indicating that BMSCsExo inhibit SCI-induced NF- $\kappa \mathrm{B}$ subunits.

There are limitations to this study that should be noted. The specific mechanisms through which exosomes downregulate complement transcription levels clearly require further investigation. Whether miRNAs or other substances that downregulate complement mRNA are carried within exosomes also needs to be established. Finally, our study was limited to changes in the complement system as determined during early stages of SCI. Whether such changes in complement are present at later stages requires further study.

Taken together, we demonstrate that BMSCs-Exo exert beneficial effects upon SCI. Specifically, we show that BMSCs-Exo can inhibit complement mRNA synthesis and release and inhibit activation of $\mathrm{NF}-\kappa \mathrm{B}$ signaling by binding to microglia cells. Accordingly, BMSCs-Exo may serve as a potential treatment for acute SCI, and the complement system may represent a primary target associated with the beneficial effects of this treatment.

\section{Acknowledgments}

This work was supported by research funding from the National Natural Science Foundation of China (No. 81873768 and 81671213 to Dr. Zhen Wang) and the Natural Science Foundation of Shandong Province (No. ZR2017MH120 to Dr. DaChuan Wang).

\section{Author Contributions}

All authors made substantial contributions to conception, design, acquisition of data, analysis and interpretation of data. All authors took part in drafting the article and revising it critically for important intellectual content. In addition, all authors gave final approval of the version to be published and agree to be accountable for all aspects of the work.

\section{Disclosure}

The authors report no conflicts of interest in this work.

\section{References}

1. Tessier-Lavigne M, Kolodkin A, Cold Spring Harbor Laboratory. Neuronal Guidance: The Biology of Brain Wiring: A Subject Collection from Cold Spring Harbor Perspectives in Biology. Cold Spring Harbor (NY): Cold Spring Harbor Laboratory Press; 2011.

2. Huang JH, Yin XM, Xu Y, et al. Systemic administration of exosomes released from mesenchymal stromal cells attenuates apoptosis, inflammation, and promotes angiogenesis after spinal cord injury in rats. $J$ Neurotrauma. 2017;34(24):3388-3396. doi:10.1089/neu.2017.5063

3. Qu J, Zhang H. Roles of mesenchymal stem cells in spinal cord injury. Stem Cells Int. 2017;2017:5251313. doi:10.1155/2017/5251313
4. Bao CS, Li XL, Liu L, Wang B, Yang FB, Chen LG. Transplantation of human umbilical cord mesenchymal stem cells promotes functional recovery after spinal cord injury by blocking the expression of IL-7. Eur Rev Med Pharmacol Sci. 2018;22(19):6436-6447. doi:10.26355/eurrev_201810_16056

5. Gu Y, Zhang Y, Bi Y, et al. Mesenchymal stem cells suppress neuronal apoptosis and decrease IL-10 release via the TLR2/ NFkappaB pathway in rats with hypoxic-ischemic brain damage. Mol Brain. 2015;8(1):65. doi:10.1186/s13041-015-0157-3

6. Osaka M, Honmou O, Murakami T, et al. Intravenous administration of mesenchymal stem cells derived from bone marrow after contusive spinal cord injury improves functional outcome. Brain Res. 2010;1343:226-235. doi:10.1016/j.brainres.2010.05.011

7. Balsam LB, Wagers AJ, Christensen JL, Kofidis T, Weissman IL, Robbins RC. Haematopoietic stem cells adopt mature haematopoietic fates in ischaemic myocardium. Nature. 2004;428(6983):668-673. doi: $10.1038 /$ nature 02460

8. Simpson RJ, Jensen SS, Lim JW. Proteomic profiling of exosomes: current perspectives. Proteomics. 2008;8(19):4083-4099. doi:10.1002/ pmic.200800109

9. Zhang B, Wang M, Gong A, et al. HucMSC-exosome mediated-Wnt4 signaling is required for cutaneous wound healing. Stem Cells. 2015;33(7):2158-2168. doi:10.1002/stem.1771

10. Cai S, Cheng X, Pan X, Li J. Emerging role of exosomes in liver physiology and pathology. Hepatol Res. 2017;47(2):194-203. doi:10.1111/hepr.12794

11. Jia H, Liu W, Zhang B, et al. HucMSC exosomes-delivered 14-3-3zeta enhanced autophagy via modulation of ATG16L in preventing cisplatininduced acute kidney injury. Am J Transl Res. 2018;10(1):101-113.

12. Gong Z, Wen M, Cheng X. Research progress on the role of exosomes in myocardial ischemia/reperfusion injury. Zhonghua Xin Xue Guan Bing Za Zhi. 2017;45(12):1112-1114. doi:10.3760/cma.j. issn.0253-3758.2017.12.021

13. Xin H, Wang F, Li Y, et al. Secondary release of exosomes from astrocytes contributes to the increase in neural plasticity and improvement of functional recovery after stroke in rats treated with exosomes harvested from MicroRNA 133b-overexpressing multipotent mesenchymal stromal cells. Cell Transplant. 2017;26(2):243257. doi:10.3727/096368916X693031

14. Wang L, Pei S, Han L, et al. Mesenchymal stem cell-derived exosomes reduce A1 astrocytes via downregulation of phosphorylated NFkappaB P65 subunit in spinal cord injury. Cell Physiol Biochem. 2018;50(4):1535-1559. doi:10.1159/000494652

15. Liu W, Wang Y, Gong F, et al. Exosomes derived from bone mesenchymal stem cells repair traumatic spinal cord injury by suppressing the activation of A1 neurotoxic reactive astrocytes. $J$ Neurotrauma. 2018;36:469-484.

16. Hao M, Ji XR, Chen H, et al. Cell cycle and complement inhibitors may be specific for treatment of spinal cord injury in aged and young mice: transcriptomic analyses. Neural Regen Res. 2018;13(3):518-527.

17. Guo Q, Li S, Liang Y, et al. Effects of $\mathrm{C} 3$ deficiency on inflammation and regeneration following spinal cord injury in mice. Neurosci Lett. 2010;485(1):32-36. doi:10.1016/j.neulet.2010.08.056

18. Li L, Xiong ZY, Qian ZM, et al. Complement C5a is detrimental to histological and functional locomotor recovery after spinal cord injury in mice. Neurobiol Dis. 2014;66:74-82. doi:10.1016/j.nbd.2014.02.008

19. Karasu E, Eisenhardt SU, Harant J, Huber-Lang M. Extracellular vesicles: packages sent with complement. Front Immunol. 2018;9:721. doi:10.3389/fimmu.2018.00721

20. Kosanovic M, Jankovic M. Isolation of urinary extracellular vesicles from Tamm- Horsfall protein-depleted urine and their application in the development of a lectin-exosome-binding assay. Biotechniques. 2014;57(3):143-149. doi:10.2144/000114208

21. Basso DM, Beattie MS, Bresnahan JC. A sensitive and reliable locomotor rating scale for open field testing in rats. J Neurotrauma. 1995;12(1):1-21. doi:10.1089/neu.1995.12.1 
22. Ross PL, Huang YN, Marchese JN, et al. Multiplexed protein quantitation in Saccharomyces cerevisiae using amine-reactive isobaric tagging reagents. Mol Cell Proteomics. 2004;3(12):1154-1169. doi:10.1074/mcp.M400129-MCP200

23. Barnum SR. Complement: A primer for the coming therapeutic revolution. Pharmacol Ther. 2017;172:63-72. doi:10.1016/j.pharmthera. 2016.11.014

24. Qiao F, Atkinson C, Song H, Pannu R, Singh I, Tomlinson S. Complement plays an important role in spinal cord injury and represents a therapeutic target for improving recovery following trauma. Am J Pathol. 2006;169(3):1039-1047. doi:10.2353/ajpath.2006.060248

25. Li L, Li J, Zhu Y, Fan G. Ephedra sinica inhibits complement activation and improves the motor functions after spinal cord injury in rats. Brain Res Bull. 2009;78(4-5):261-266. doi:10.1016/j. brainresbull.2008.10.002

26. Anderson AJ, Najbauer J, Huang W, Young W, Robert S. Upregulation of complement inhibitors in association with vulnerable cells following contusion-induced spinal cord injury. J Neurotrauma. 2005;22(3):382-397. doi:10.1089/neu.2005.22.382

27. Anderson AJ, Robert S, Huang W, Young W, Cotman CW. Activation of complement pathways after contusion-induced spinal cord injury. $J$ Neurotrauma. 2004;21(12):1831-1846. doi:10.1089/neu.2004.21.1831

28. Rebhun J, Botvin J. Complement elevation in spinal cord injury. Ann Allergy. 1980;44(5):287-288.

29. Ohlsson M, Havton LA. Complement activation after lumbosacral ventral root avulsion injury. Neurosci Lett. 2006;394(3):179-183. doi:10.1016/j.neulet.2005.10.037

30. Nguyen HX, Galvan MD, Anderson AJ. Characterization of early and terminal complement proteins associated with polymorphonuclear leukocytes in vitro and in vivo after spinal cord injury. $J$ Neuroinflammation. 2008;5:26. doi:10.1186/1742-2094-5-26

31. Qiao F, Atkinson C, Kindy MS, et al. The alternative and terminal pathways of complement mediate post-traumatic spinal cord inflammation and injury. Am J Pathol. 2010;177(6):3061-3070. doi:10.2353/ ajpath.2010.100158

32. Galvan MD, Luchetti S, Burgos AM, et al. Deficiency in complement C1q improves histological and functional locomotor outcome after spinal cord injury. J Neurosci. 2008;28(51):13876-13888. doi:10.1523/JNEUR OSCI.2823-08.2008

33. Juliano SL, Friedman DP, Eslin DE. Corticocortical connections predict patches of stimulus-evoked metabolic activity in monkey somatosensory cortex. J Comp Neurol. 1990;298(1):23-39. doi:10.1002/cne.902980103

34. Toh WS, Zhang B, Lai RC, Lim SK. Immune regulatory targets of mesenchymal stromal cell exosomes/small extracellular vesicles in tissue regeneration. Cytotherapy. 2018;20:1419-1426. doi:10.1016/j. jcyt.2018.09.008

35. Eirin A, Zhu XY, Puranik AS, et al. Integrated transcriptomic and proteomic analysis of the molecular cargo of extracellular vesicles derived from porcine adipose tissue-derived mesenchymal stem cells. PLoS One. 2017;12(3):e0174303. doi:10.1371/journal.pone.0174303
36. Karpman D, Stahl AL, Arvidsson I, et al. Complement interactions with blood cells, endothelial cells and microvesicles in thrombotic and inflammatory conditions. Adv Exp Med Biol. 2015;865:19-42. doi:10.1007/978-3-319-18603-0_2

37. Unnewehr H, Rittirsch D, Sarma JV, et al. Changes and regulation of the C5a receptor on neutrophils during septic shock in humans. $J$ Immunol. 2013;190(8):4215-4225. doi:10.4049/jimmunol.1200534

38. Curry N, Raja A, Beavis J, Stanworth S, Harrison P. Levels of procoagulant microvesicles are elevated after traumatic injury and platelet microvesicles are negatively correlated with mortality. $J$ Extracell Vesicles. 2014;3:25625. doi:10.3402/jev.v3.24384

39. Clayton A, Harris CL, Court J, Mason MD, Morgan BP. Antigenpresenting cell exosomes are protected from complement-mediated lysis by expression of CD55 and CD59. Eur J Immunol. 2003;33 (2):522-531. doi:10.1002/immu.200310028

40. Robbins PD, Morelli AE. Regulation of immune responses by extracellular vesicles. Nat Rev Immunol. 2014;14(3):195-208. doi:10.1038/ nri3622

41. Knickelbein JE, Liu B, Arakelyan A, et al. Modulation of immune responses by extracellular vesicles from retinal pigment epithelium. Invest Ophthalmol Vis Sci. 2016;57(10):4101-4107. doi:10.1167/ iovs.15-18353

42. Xu C, Fu F, Li X, Zhang S. Mesenchymal stem cells maintain the microenvironment of central nervous system by regulating the polarization of macrophages/microglia after traumatic brain injury. Int J Neurosci. 2017;127(12):1124-1135. doi:10.1080/00207454.2017.1325884

43. Jin X, Yamashita T. Microglia in central nervous system repair after injury. J Biochem. 2016;159(5):491-496. doi:10.1093/jb/mvw009

44. Long Q, Upadhya D, Hattiangady B, et al. Intranasal MSC-derived A1-exosomes ease inflammation, and prevent abnormal neurogenesis and memory dysfunction after status epilepticus. Proc Natl Acad Sci US A. 2017;114(17):E3536-E3545. doi:10.1073/pnas.1703920114

45. Perets N, Betzer O, Shapira R, et al. Golden exosomes selectively target brain pathologies in neurodegenerative and neurodevelopmental disorders. Nano Lett. 2019;19:3422-3431. doi:10.1021/acs. nanolett. $8 \mathrm{~b} 04148$

46. Woodruff TM, Tenner AJ. A commentary on: "NFkappaB-activated astroglial release of complement $\mathrm{C} 3$ compromises neuronal morphology and function associated with Alzheimer's Disease". A cautionary note regarding C3aR. Front Immunol. 2015;6:220. doi:10.3389/ fimmu.2015.00220

47. Lian H, Yang L, Cole A, et al. NFkappaB-activated astroglial release of complement C3 compromises neuronal morphology and function associated with Alzheimer's disease. Neuron. 2015;85(1):101-115. doi:10.1016/j.neuron.2014.11.018

48. Barton PA, Warren JS. Complement component C5 modulates the systemic tumor necrosis factor response in murine endotoxic shock. Infect Immun. 1993;61(4):1474-1481.

49. Brambilla R, Bracchi-Ricard V, Hu WH, et al. Inhibition of astroglial nuclear factor kappaB reduces inflammation and improves functional recovery after spinal cord injury. J Exp Med. 2005;202(1):145-156. doi:10.1084/jem.20041918

\section{Publish your work in this journal}

Drug Design, Development and Therapy is an international, peerreviewed open-access journal that spans the spectrum of drug design and development through to clinical applications. Clinical outcomes, patient safety, and programs for the development and effective, safe, and sustained use of medicines are a feature of the journal, which has also been accepted for indexing on PubMed Central. The manuscript management system is completely online and includes a very quick and fair peer-review system, which is all easy to use. Visit http://www. dovepress.com/testimonials.php to read real quotes from published authors. 